\title{
Audio Transmission using Li-Fi Technology
}

\author{
Bolli Jagadeeswari, Charapu Sai Anusha, Dangeti Monisa, Medisetti Preethi
}

\author{
Department of Electrical and Electronics Engineering, \\ Pragati Engineering College, Surampalem, Andhra Pradesh, India
}

\begin{abstract}
How to cite this paper: Bolli Jagadeeswari | Charapu Sai Anusha | Dangeti Monisa | Medisetti Preethi "Audio Transmission using Li-Fi Technology" Published in International Journal of Trend in Scientific Research and Development (ijtsrd), ISSN: 24566470, Volume-3 | Issue-3, April 2019, pp.1008-1011, URL: https://www.ijtsrd.c om/papers/ijtsrd23 156.pdf

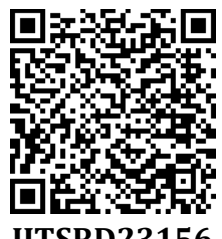

Copyright (C) 2019 by author(s) and International Journal of Trend in Scientific Research and Development Journal. This is an Open Access article distributed under the terms of the Creative Commons

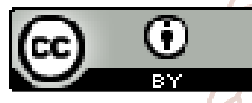
Attribution License (CC BY 4.0) (http://creativecommons.org/licenses/ by/4.0)

\section{INTRODUCTION}

With the boom of Smart Phones, Internet of things, Industrial Automations, Smart phone Automation systems etc. the demand for internet is also growing exponentially. The technology has evolved so much that everything from our car to our refrigerator needs a connection to the internet. This raises other questions like; will there be enough bandwidth for all these devices? Will these data be secure? Will the existing system be fast enough for all these data? Will there be too much conjunction on network traffic?

In today world, communication between the devices is much common. Radio wave spectrum is very small part of spectrum available for communication. Wi-Fi and Bluetooth are currently the two prominent short range wireless technologies but with increase in advanced technology and number of user the network becomes overloaded which results in failure to provide high data rate. All these questions will be tackled by this upcoming technology called Li-Fi.. So what is Li-Fi? The term Li-Fi stands for "Light Fidelity". This is believed to be the next generation of internet, where Light will be used as a medium to transport data.

Li-Fi is transmission of data using visible light by sending data through an LED light bulb that varies in intensity faster than the human eye can follow. The idea of Li-Fi was introduced by a German physicist Harald Hass, which he also referred to as "Data through illumination". The general term visible light communication includes any use of the visible light portion of the electromagnetic spectrum to transmit information. Haas promoted this technology in his 2011 TED Global talk and helped start a company to market it. Pure Li-Fi, formerly pure VLC, is an original equipment manufacturer (OEM) firm set up to commercialize $\mathrm{Li}-\mathrm{Fi}$ products for integration with existing LED-lighting systems .According to Hass, the light, which he referred to as 'DLight' , can be used to produce data rates higher than 1 Giga bits per second which is much faster than our average broadband connection.

\section{WORKING OF Li-Fi}

LEDs can be switched on and off faster than the human eye can detect since the operating speed of LEDs is less than $1 \mu$ s,thereby causing the light source to appear to be continuously on .This invisible on-off activity enables data transmission using binary codes .Switching on an LED is binary ' 1 ', switching it off is binary ' 0 '. It is possible to encode data in light by varying the rate at which LEDs flicker on and off to give different strings of $1 \mathrm{~s}$ and 0s.Modulation is so rapid that humans cannot notice it A light sensitive device(photo detector) then receives the signal and converts it back into original data. 


\section{EXISTING SYSTEM}

Transmitting data through photodiodes has been happening for a long time through our IR Remotes. Every time we pressed a button on our Television remote the IR LED in the Remote pulses very fast this will be received by the Television and then decoded for the information. But, this old method is very slow and cannot be used to transmit any worthy data.

Wi-Fi and Bluetooth are the two consistent wide range sources used by multiple applications today. However, these methods use radio frequency spectrum and noise of the signal is very high. Other inconveniences of these methods are special equipment requirement, high power consumption, and high cost. Secure data transmission is not available here.WPS key encryption can be provided and hacking can also be done easily since it uses radio wave transmission it is harmful to health.

\section{PROPOSED SYSTEM}

Visible light is a new technique of data transmission method. $\mathrm{Li}-\mathrm{Fi}$, data is transmitted by modulating the intensity of the light, which is then received by a Photo-sensitive detector.VLC, consists of a light source as a transmitter and detector as a receiver. Louder the voice, the glow of the LED will be more. The receiver section interprets the incoming light which is detected using a solar panel and converts to the audible sound signal with the help of Speaker. Hence with Li-Fi this method is made sophisticated by using more than one LED and passing more than one data stream at a given time. This way more information can be passed and hence a faster data communication is possible.

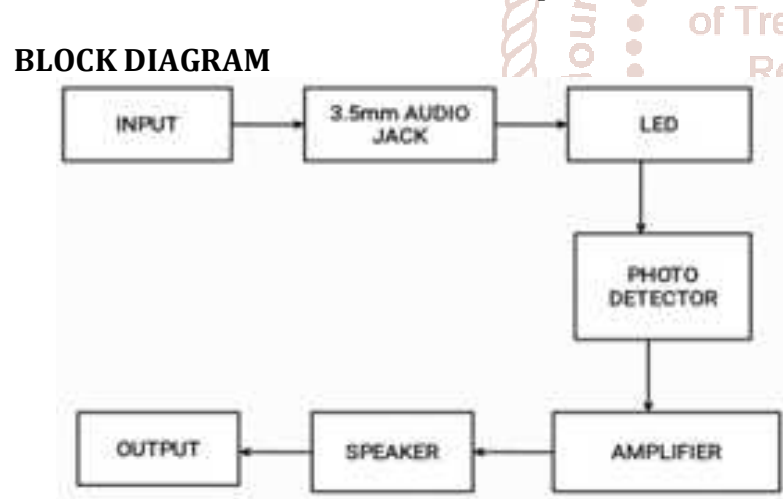

Fig 1: Block diagram of proposed system

In the process of an audio communication through the visible light, on the transmitter side an audio signal (any music from mobile) is used as the input signal. This signal is fed to the LED. The light signal from the LED varies according to the intensity of an audio signal. At the receiver side light dependent resister will receive the light signal and correspondingly generate an electrical signal proportional to it. This electrical signal is processed by a low voltage audio power amplifier, which is then fed to a speaker and it produces the audio signal which was at the input of the transmitter side.

\section{HARDWARE REQUIREMENTS}

\section{INPUT:}

Input consists of analog signal, which is usually taken from the audio output of the Mobile Phone, Laptop or any other musical Instruments. The signal will be at low voltage level which is not enough to drive an LED, So in order to drive the LEDs we have to amplify the signal using amplifiers.

\section{AUDIO JACK:}

It is a Mobile connector used to connect the mobile phones with the audio device.

Here it is used to connect the mobile device or any other device like IPAD, MP3 Player, etc. with Li-Fi as input audio signal.

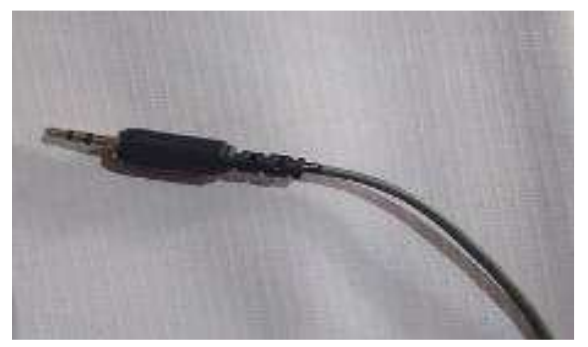

Fig2:3.5mm Audio Jack

\section{LEDs:}

In Li-Fi Transmission, the most important requirement of light source is its ability to turn ON and OFF Repeatedly in very short intervals (in ns range). So we use LEDs which have very low switching time. These LEDs turn ON and OFF in nanosecond based on the Pulse signal. Since the switching taking at a faster rate, it cannot be detected by Human eye. So it will appear as illuminating even though they are blinking. Thus modulated signal is transmitted to receiver via Visible Light.

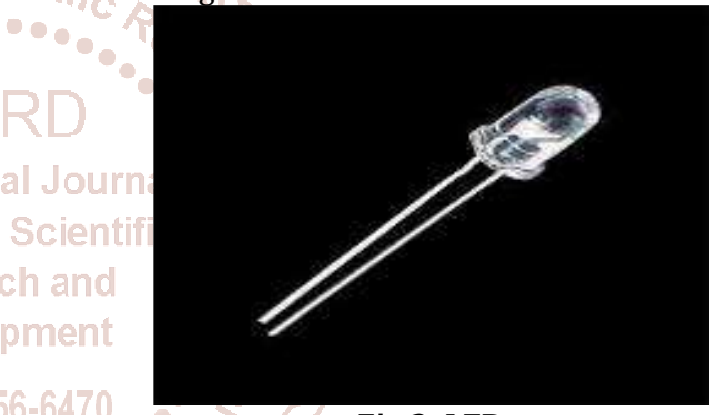

Fig 3: LED

\section{PHOTO DETECTOR:}

The transmitted signal from the LEDs has to be detected, demodulated and acknowledged. So in order to detect the message signal from the blinking LED light, we use a photo cell or a Solar Cell (which comprises large no of photo cells connected in series).

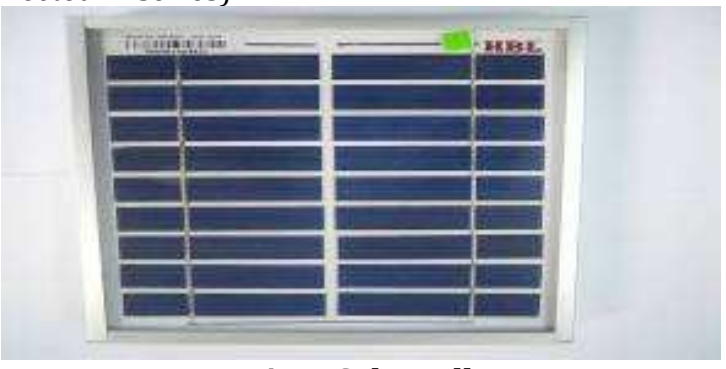

Fig 4: Solar cell

The solar cell detects only the variation of the light, since the blinking can be easily detected and output of the solar cell will be the message signal in the analog form. So using solar we could detect and demodulate the message signal transmitted.

\section{AMPLIFIER AND SPEAKER:}

The demodulated signal will be at low voltage range. So it is amplified to the arbitrary voltage level using an amplifier. This amplifier will be same type of amplifier which we used 
in transmitter side. This is due to the fact that if any phase errors occurred, it will be cleared at this stage. The speaker will convert the electrical signal to the audible form using electro magnets present in the speaker.

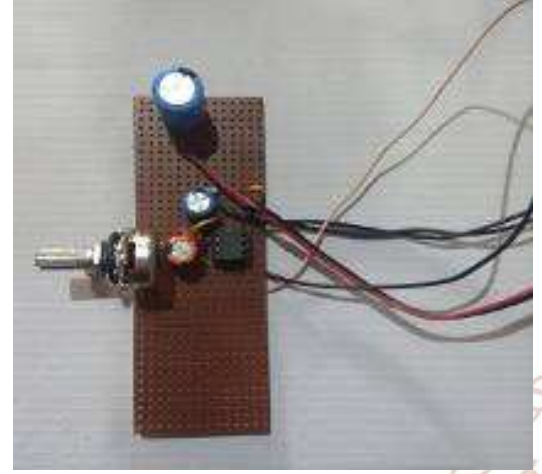

Fig 5: Amplifier
$>$ Li-Fi can also be used in traffic management system for reducing the number of accidents by vehicle to vehicle communication.

$>$ Sensitive data - Hospitals are an environment where both EMI sensitivity and security of data are issues. Li-Fi can enable the better disposition of secure networked medical devices, patient accounts, etc.

$>$ The system can be used in offices, hotels, auditoria, etc, where bright lighting is required throughout the day.

$>$ Transmit audio signals from a microphone on the dais to speakers in an auditorium using pre-installed LED lights.

\section{RESULT}

The transmission of audio signal was done through a Smartphone at the transmitter end, providing the audio signal through the $3.5 \mathrm{~mm}$ jack. The $3.5 \mathrm{~mm}$ audio jack and the input audio from the phone is converted from digital to analog. A typical $3.5 \mathrm{~mm}$ audio jack has three output lines namely, right, left and the ground. The left and right have the audio output signal, which is connected to the negative of the $9 \mathrm{~V}$ battery. The ground of the $3.5 \mathrm{~mm}$ jack is given to the negative of the LED array connected on a breadboard and the positive of the $9 \mathrm{~V}$ array is given to the resistors in series with the LED array. This circuit effectively modulates the intensity of the LEDs light, which acts as carrier wave, according to the effective voltage difference. The fluctuations occur at a high speed, invisible to the naked human eye. This variation in the intensity of light, however, is captured on a solar panel that acts as a photo detector. It captures all the variations and sends the received signal to the amplifier, which amplifies the signal and giving the audio output through the speaker. The sound intensity received from the speaker varies based on the distance of the solar panel from the LED arrays. This shows that the information can be received from the line of sight of the LED array. As the distance between LED array and the solar panel increases, the intensity of light reduces and the light becomes more scattered thus, making it difficult for the solar panel to detect all the light rays being emitted.

The demodulated audible signal is transmitted from speaker to its final destination. So, the audience can listen to the message that has been transmitted from the source.

\section{ADVANTAGES}

$>$ Faster Data Transmission than Wi-Fi.

$>$ Easy and Inexpensive to Deploy.

$>$ Security Due to the Limitations of Light.

$>$ Immune from Electromagnetic interferences.

$>$ Low Cost

$>$ Portability

$>$ Low bit error rate

$>$ High efficiency

$>$ Consumes less energy

\section{APPLICATIONS}

$>$ Li-Fi can be used in various areas like Hospitals

$>$ Automation because operating rooms do not allow Wi$\mathrm{Fi}$, since $\mathrm{Wi}-\mathrm{Fi}$ radiates harmful signals.

$>$ Li-Fi audio transmission can be used in petrochemical industries automations where use of radio spectrum is very dangerous.

$>$ Li-Fi can also be used in Power plants as Wi-Fi and many other radiation types are very bad for such sensitive areas.

$>$ Li-Fi can also be used in underwater systems for audio communications and device control

$>$ Localised advertising can be done by broadcasting through the Li-Fi channel into smaller distances.

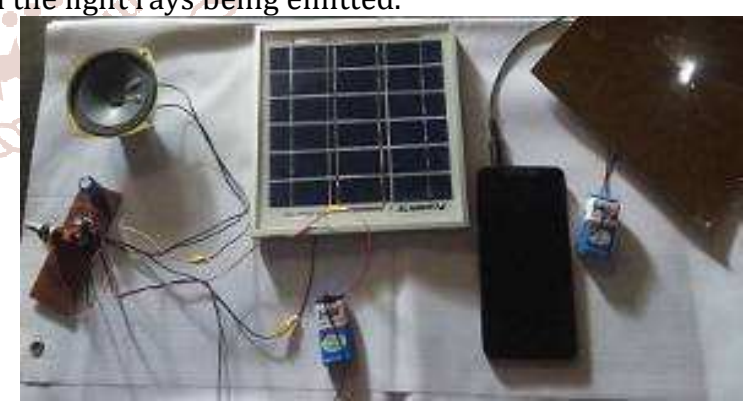

\section{CONCLUSION}

The possibilities are numerous and can be explored further. If his technology can be put into practical use, every bulb can be used something like a Wi-Fi hotspot to transmit wireless data and we will proceed toward the cleaner, greener, safer and brighter future. The concept of Li-Fi is currently attracting a great deal of interest, not least because it may offer a genuine and very efficient alternative to radio-based wireless. As a growing number of people and their many devices access wireless internet, the airwaves are becoming increasingly clogged, making it more and more difficult to get a reliable, high-speed signal. This may solve issues such as the shortage of radio-frequency bandwidth and also allow internet where traditional radio based wireless isn't allowed such as aircraft or hospitals.
Fig 7: Overall view of the project 


\section{FUTURE SCOPE}

$\mathrm{Li}-\mathrm{Fi}$ is an emerging technology and hence it has vast potential. A lot of research can be conducted in this field. Already, a lot of scientists are involved in extensive research in this field. The future of Li-Fi is Gi-Fi. Gi-Fi or gigabit wireless refers to wireless communication at a data rate of more than one billion bits (gigabit) per second.

By using Li-Fi we can have Energy saving Parallelism. In future we can have LED array beside a motorway helping to light the road, displaying the latest traffic updates and transmitting internet information to wirelessly to passengers Laptops, Notebooks and Smart phones. This is the kind of extra ordinary, energy saving parallelism that is believed to deliver by this pioneering technology.

Further research in the field can look into the following issues:

1. Driving illumination grade LEDs at high speed.

2. Increasing data rate with parallelism/arrays.

3. Achieving low complexity/low cost modulation.

4. Overcoming the line of sight constraint.

5. Achieving seamless interoperability with other networks.

6. Making Li-Fi work in environments with little or no light.

\section{ACKNOWLEDGEMENT}

\section{REFERENCES}

[1] Prof. Amit K. et al., "Li-Fi: Wireless Communication Media, " International Journal of Innovative Research in Electrical, Electronics, instrumentation and control engineering vol.4, Feb., 2016.

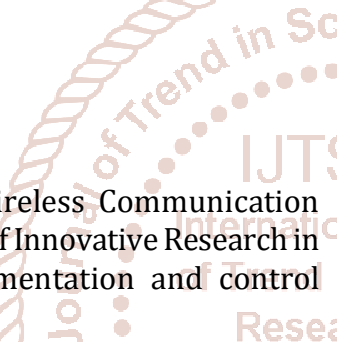

[2] J. M. Kahn and J. R. Barry, "Wireless Infrared Communications, " Proceedings of the IEEE, pp. 265298, 1997. doi:10.1109/5.554222

[3] T. Komine and M. Nakagawa, "Fundamental analysis of visible light communication systems using LED Lights,"
IEEE Trans. Consumer Electronics, vol. 59, no.1, Feb 2004.

[4] R. Mahendran PG Scholar Embedded System Technology S. A Engineering College, Chennai. "Integrated Li-Fi (Light Fidelity) For Smart Communication through Illumination", 2016 International Conference on Advanced Communication Control and Computing Technologies (ICACCCT).

[5] Vega, Anaa (14 July 2014). "Li-Fi record data transmission of 10GBps set using LED lights". Engineering and Technology Magazine. Retrieved 15 Feb. 2015.

[6] Haas, Harald (July 2011). "Wireless data from every light bulb". TED Global. Edinburgh, Scotland.

[7] Li-Fi internet solution from Russian company attracting foreign clients, Russia and India Report, Russia beyond the Headlines, 1 July 2014.

[8] "What is Li-Fi" (2014), [Online]. Available: http://purelifi.com/what_is_li-fi/li-fi-features/

[9] P. Amirshahi, M. Kavehrad, "Broadband Access over Medium and Low Voltage Power lines and use of White Light Emitting Diodes for Indoor Communications, " IEEE Consumer.

[10] M. Kavehrad, "Sustainable Energy-Efficient Wireless Applications Using Light, " IEEE Commune. Mag., vol. 48, no. 12 , Dec. 2010 , pp. 66- 73.

[11] Haas, Harald (19 April 2013). "High-speed wireless networking using visible light".

[12] "A Visible Light Communication System for Indoor Application" Amrutha. S, Ansu Mathew, Rajasree. R, Swarthy Sugathan; Aravind. S, International Journal of Engineering and Innovative Technology (IJEIT) Volume 3 , Issue 12 , June 2014. 